\title{
Synthesis and antimicrobial studies of some Mannich bases carrying imidazole moiety
}

\author{
PRIYA V. FRANK ${ }^{1 *}$ \\ MAHESHA MANJUNATHA POOJARY ${ }^{1}$ \\ NARAL DAMODARA 1 \\ CHANDRASHEKHAR CHIKKANNA ${ }^{2}$ \\ ${ }^{1}$ Department of Chemistry, Canara \\ Engineering College, Benjanapadavu \\ Mangalore-574219, Karnataka, India \\ ${ }^{2}$ PG Department of Medicinal Chemistry \\ SDM College, Ujire-574240, Karnataka \\ India
}

Accepted January 17, 2013

\begin{abstract}
Starting from 2-methyl-4-nitro-imidazole, new 5-(2-methyl-4-nitro-1-imidazomethyl)-1,3,4-oxadiazole-2-thione (3) was synthesized and was subjected to Mannich reaction with appropriate amines to yield a new series of 3-substituted aminomethyl-5-(2-methyl-4-nitro-1-imidazomethyl)-1,3,4-oxadiazole-2-thiones (4a-j). The structure of the title compounds was elucidated by elemental analysis and spectral data. The newly synthesized Mannich bases were screened for their antibacterial and antifungal activity. Many of these compounds exhibited potent antifungal activity.

Keywords: imidazole derivatives, Mannich bases, oxadiazole, antibacterial activity, antifungal activity
\end{abstract}

The most important applications of Mannich bases are in the field of pharmaceutical products $(1,2)$. Studies have revealed that Mannich bases show good anticancer (3), antimycobacterial (4), remarkable anti-HIV and antitubercular activities (5). The presence of the basic Mannich side-chain has shown marked antimalarial (6), anti-inflammatory, analgesic $(7,8)$ and antimicrobial activities $(9)$. Imidazole nucleus is also a major component of a variety of drugs such as angiotensin II receptor antagonists, oral anti-inflammatory agents, protein kinase inhibitors and fungicides (10). It is frequently found as a part of a large number of biologically and medicinally significant substances (11, 12).

There are not many reports describing the synthesis and biological studies of Mannich bases bearing imidazole moiety. From this point of view, the present study successfully describes a general and convenient method for the synthesis of a series of 3-substituted aminomethyl-5-(2-methyl-4-nitro-1-imidazomethyl)-1,3,4-oxadiazole-2-thiones and depicts the antimicrobial activity of the newly synthesized Mannich bases.

\footnotetext{
* Correspondence; e-mail: priyafrank@gmail.com
} 


\section{EXPERIMENTAL}

Melting points were determined in open glass capillaries using a VEEGO, VMP-D digital melting point apparatus (Veego, India) and are uncorrected. Elemental analyses were performed on a Perkin-Elmer 2400 analyzer (Perkin-Elmer, USA) and were found within $\pm 0.4 \%$ of the theoretical values. The IR spectra were recorded as $\mathrm{KBr}$ discs using a JASCO FTIR 4100 spectrometer (Jasco, Japan). ${ }^{1} \mathrm{H}$ NMR spectra were recorded on a Bruker Avance II 400 NMR spectrometer (Bruker Instruments Inc., USA) using $\mathrm{CDCl}_{3}$ or DMSO- $d_{6}$ as solvent and TMS as internal standard. Mass spectra were measured on a JEOL GCMATE II mass spectrometer (Jeol, Japan).

2-Methyl-4-nitro imidazole, ethyl choloroacetate, ciprofloxacin and fluconazole were procured from Sigma-Aldrich, USA. All other chemicals used in the study were of analytical grade.

\section{Synthetic procedures}

2-Methyl-4-nitro-1-imidazo-ethyl acetate (1). - A mixture of 2-methyl-4-nitro-imidazole $(12.7 \mathrm{~g}, 0.1 \mathrm{~mol})$, ethyl chloroacetate $(10.7 \mathrm{~mL}, 0.1 \mathrm{~mol})$ and potassium carbonate $(20.4 \mathrm{~g}$, $0.15 \mathrm{~mol})$ in dry acetone $(200 \mathrm{~mL})$ was refluxed for $50 \mathrm{~h}$. The reaction mixture was filtered hot and the solvent was distilled off from the filtrate. The crude ester $\mathbf{1}$ thus obtained was purified by recrystallization from absolute ethanol.

2-Methyl-4-nitro-1-imidazo-acethydrazide (2). - A mixture of ester (1) (21.3 g, $0.1 \mathrm{~mol})$ and $99 \%$ hydrazine hydrate $(4.9 \mathrm{~mL}, 0.1 \mathrm{~mol})$ in absolute ethanol $(50 \mathrm{~mL})$ was refluxed for $8 \mathrm{~h}$. On cooling, the solution gave a solid mass of hydrazide 2 , which was collected by filtration, and recrystallized from absolute ethanol.

5-(2-Methyl-4-nitro-1-imidazomethyl)-1,3,4-oxadiazole-2-thione (3). - A mixture of 2-methyl-4-nitro-1-imidazo-acethydrazide (2) (19.9 g, $0.1 \mathrm{~mol}), \mathrm{KOH}(5.6 \mathrm{~g}, 0.1 \mathrm{~mol})$, carbon disulphide $(6.0 \mathrm{~mL}, 0.1 \mathrm{~mol})$ in absolute ethanol $(100 \mathrm{~mL})$ was placed in a round-bottomed flask fitted with a water cooled condenser and refluxed on a water bath till the evolution of hydrogen sulphide ceased. The excess of alcohol was removed by distillation. The reaction mixture was cooled to room temperature and the contents were poured to ice-cold water and neutralized with dilute hydrochloric acid. The solid precipitated was filtered, washed thoroughly with water and dried. The product was further purified by recrystallization from the ethanol-dioxane mixture (1:1) to give 3 .

3-Substituted aminomethyl-5-(2-methyl-4-nitro-1-imidazomethyl)-1,3,4-oxadiazole-2-thiones $(4 a-j)$. - A mixture of the solution of 5-(2-methyl-4-nitro-1-imidazomethyl)-1,3,4-oxadiazole-2-thione (3) $(2.41 \mathrm{~g}, 0.01 \mathrm{~mol})$ in absolute ethanol and dioxane mixture $(1: 1,20 \mathrm{~mL})$ was treated with formaldehyde $(40 \%, 1.5 \mathrm{~mL}, 0.05 \mathrm{~mol})$. Later, the appropriate amine $(0.01 \mathrm{~mol})$ in absolute ethanol $(10 \mathrm{~mL})$ was added with stirring and the reaction mixture was stirred overnight. The precipitated Mannich base was collected by filtration and dried. Recrystallization was done from the ethanol-DMF mixture (1:1) to give compounds $4 \mathbf{a}-\mathbf{j}$.

\section{Antimicrobial studies}

The antimicrobial activity of the newly synthesized Mannich bases was checked against three fungal strains, viz., Candida albicans, Trichophyton rubrum and Trichophyton 
mentagrophytes and two pathogenic bacteria, viz., Staphylococcus aureus and Streptococcus pyogenes. Fluconazole and ciprofloxacin were used as standards. The synthesized compounds and standards were dissolved in DMSO to give a concentration of $1000 \mu \mathrm{g} \mathrm{mL}-1$. The minimum inhibitory concentration $(M I C)$ was determined by the two-fold tube dilution method (13). Dilutions of test and standard compounds were prepared in Sabouraud dextrose broth for fungi and nutrient broth for bacteria. The samples were incubated at $25{ }^{\circ} \mathrm{C}$ for 48 hours for C. albicans, $5-7$ days at $20-22{ }^{\circ} \mathrm{C}$ for T. rubrum and T. mentagrophytes and at $37^{\circ} \mathrm{C}$ for 24 hours for bacteria. At the end of the incubation period, MIC values were recorded as the lowest concentrations of substances that showed no visible turbidity.

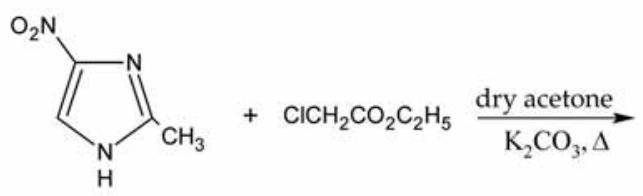<smiles>CCOCn1cc([N+](=O)[O-])nc1C</smiles><smiles>Cc1nc([N+](=O)[O-])cn1Cc1n[nH]c(=S)o1</smiles><smiles>CC(=O)OO</smiles><smiles>CCON=CCn1cc([N+](=O)[O-])nc1C</smiles><smiles>[R10]NCn1nc(Cn2cc([N+](=O)[O-])nc2C)oc1=S</smiles>

Scheme 1. 


\section{RESULTS AND DISCUSSION}

\section{Chemistry}

In the present work, a series of Mannich bases has been synthesized. Elimination reaction of 2-methyl-4-nitro- $1 \mathrm{H}$-imidazole with ethyl chloroacetate in the presence of po-

Table I. Physical and analytical properties of the new compounds

\begin{tabular}{|c|c|c|c|c|c|c|c|}
\hline \multirow[t]{2}{*}{ Compd. } & \multirow[t]{2}{*}{$\mathrm{R}_{1}$} & \multirow[t]{2}{*}{$\mathrm{R}_{2}$} & \multirow{2}{*}{$\begin{array}{l}\text { Molecular formula } \\
\qquad\left(M_{\mathrm{r}}\right)\end{array}$} & \multirow{2}{*}{$\begin{array}{l}\text { Yield }(\%) \\
\text { M. p. }\left({ }^{\circ} \mathrm{C}\right)\end{array}$} & \multicolumn{3}{|c|}{$\begin{array}{c}\text { Elemental analysis }(\%) \\
\text { Found/calcd. }\end{array}$} \\
\hline & & & & & C & $\mathrm{H}$ & $\mathrm{N}$ \\
\hline 1 & - & - & $\begin{array}{c}\mathrm{C}_{8} \mathrm{H}_{11} \mathrm{~N}_{3} \mathrm{O}_{4} \\
(213.19)\end{array}$ & $\begin{array}{c}80 \\
109-111\end{array}$ & $\begin{array}{r}45.05 / \\
45.03\end{array}$ & $\begin{array}{c}5.15 / \\
5.16\end{array}$ & $\begin{array}{r}19.69 / \\
19.70\end{array}$ \\
\hline 2 & - & - & $\begin{array}{c}\mathrm{C}_{6} \mathrm{H}_{9} \mathrm{~N}_{5} \mathrm{O}_{3} \\
(199.17)\end{array}$ & $\begin{array}{c}66 \\
197-199\end{array}$ & $\begin{array}{c}36.17 / \\
36.15\end{array}$ & $\begin{array}{c}4.53 / \\
4.52\end{array}$ & $\begin{array}{r}35.13 / \\
35.15\end{array}$ \\
\hline 3 & - & - & $\begin{array}{c}\mathrm{C}_{7} \mathrm{H}_{7} \mathrm{~N}_{5} \mathrm{O}_{3} \mathrm{~S} \\
(241.24)\end{array}$ & $\begin{array}{c}65 \\
242-243\end{array}$ & $\begin{array}{r}34.82 / \\
34.83\end{array}$ & $\begin{array}{c}2.90 / \\
2.91\end{array}$ & $\begin{array}{r}29.02 / \\
29.04\end{array}$ \\
\hline $4 a$ & $\mathrm{H}$ & $\mathrm{C}_{6} \mathrm{H}_{5}$ & $\begin{array}{c}\mathrm{C}_{14} \mathrm{H}_{14} \mathrm{~N}_{6} \mathrm{O}_{3} \mathrm{~S} \\
(346.36)\end{array}$ & $\begin{array}{c}87 \\
150-152\end{array}$ & $\begin{array}{r}48.52 / \\
48.50\end{array}$ & $\begin{array}{r}4.03 / \\
4.04\end{array}$ & $\begin{array}{r}24.24 / \\
24.25\end{array}$ \\
\hline $4 b$ & $\mathrm{H}$ & $\begin{array}{c}\mathrm{C}_{6} \mathrm{H}_{4} \mathrm{C}_{2} \mathrm{H}_{5}- \\
p\end{array}$ & $\begin{array}{c}\mathrm{C}_{16} \mathrm{H}_{18} \mathrm{~N}_{6} \mathrm{O}_{3} \mathrm{~S} \\
(374.42)\end{array}$ & $\begin{array}{c}63 \\
136-137\end{array}$ & $\begin{array}{r}51.29 / \\
51.28\end{array}$ & $\begin{array}{c}4.80 / \\
4.81\end{array}$ & $\begin{array}{r}22.43 / \\
22.44\end{array}$ \\
\hline $4 c$ & $\mathrm{H}$ & $\mathrm{C}_{6} \mathrm{H}_{4} \mathrm{~F}-p$ & $\begin{array}{c}\mathrm{C}_{14} \mathrm{H}_{13} \mathrm{~N}_{6} \mathrm{O}_{3} \mathrm{FS} \\
(364.36)\end{array}$ & $\begin{array}{c}71 \\
199-121\end{array}$ & $\begin{array}{c}46.12 / \\
46.11\end{array}$ & $\begin{array}{c}3.59 / \\
3.57\end{array}$ & $\begin{array}{r}23.04 / \\
23.05\end{array}$ \\
\hline $4 \mathrm{~d}$ & $\mathrm{H}$ & $\mathrm{C}_{6} \mathrm{H}_{4} \mathrm{Cl}-p$ & $\begin{array}{c}\mathrm{C}_{14} \mathrm{H}_{13} \mathrm{~N}_{6} \mathrm{O}_{3} \mathrm{ClS} \\
(380.81)\end{array}$ & $\begin{array}{c}65 \\
135-137\end{array}$ & $\begin{array}{r}44.11 / \\
44.12\end{array}$ & $\begin{array}{c}3.42 / \\
3.41\end{array}$ & $\begin{array}{r}22.05 / \\
22.06\end{array}$ \\
\hline $4 e$ & $\mathrm{H}$ & $\mathrm{C}_{6} \mathrm{H}_{4} \mathrm{Br}-p$ & $\begin{array}{c}\mathrm{C}_{14} \mathrm{H}_{13} \mathrm{~N}_{6} \mathrm{O}_{3} \mathrm{BrS} \\
(425.26)\end{array}$ & $\begin{array}{c}51 \\
142-144\end{array}$ & $\begin{array}{r}39.50 / \\
39.51\end{array}$ & $\begin{array}{c}3.05 / \\
3.06\end{array}$ & $\begin{array}{r}19.73 / \\
19.75\end{array}$ \\
\hline $4 \mathrm{f}$ & $\mathrm{H}$ & & $\begin{array}{c}\mathrm{C}_{15} \mathrm{H}_{15} \mathrm{~N}_{7} \mathrm{O}_{5} \mathrm{~S} \\
(405.39)\end{array}$ & $\begin{array}{c}54 \\
200-202\end{array}$ & $\begin{array}{r}44.42 / \\
44.40\end{array}$ & $\begin{array}{c}3.71 / \\
3.70\end{array}$ & $\begin{array}{r}24.18 / \\
24.17\end{array}$ \\
\hline $4 g$ & - & & $\begin{array}{c}\mathrm{C}_{20} \mathrm{H}_{18} \mathrm{~N}_{6} \mathrm{O}_{3} \mathrm{~S} \\
(422.46)\end{array}$ & $\begin{array}{c}60 \\
125-127\end{array}$ & $\begin{array}{r}56.82 / \\
56.81\end{array}$ & $\begin{array}{c}4.25 / \\
4.26\end{array}$ & $\begin{array}{r}19.87 / \\
19.88\end{array}$ \\
\hline $4 h$ & - & & $\begin{array}{c}\mathrm{C}_{12} \mathrm{H}_{17} \mathrm{~N}_{7} \mathrm{O}_{3} \mathrm{~S} \\
(339.37)\end{array}$ & $\begin{array}{c}51 \\
204-206\end{array}$ & $\begin{array}{r}42.45 / \\
42.43\end{array}$ & $\begin{array}{c}5.02 / \\
5.01\end{array}$ & $\begin{array}{r}28.86 / \\
28.88\end{array}$ \\
\hline $4 i$ & - & & $\begin{array}{c}\mathrm{C}_{13} \mathrm{H}_{19} \mathrm{~N}_{7} \mathrm{O}_{3} \mathrm{~S} \\
(353.40)\end{array}$ & $\begin{array}{c}50 \\
193-195\end{array}$ & $\begin{array}{r}44.15 / \\
44.14\end{array}$ & $\begin{array}{c}5.36 / \\
5.38\end{array}$ & $\begin{array}{r}27.72 / \\
27.73\end{array}$ \\
\hline $4 j$ & - & & $\begin{array}{c}\mathrm{C}_{14} \mathrm{H}_{21} \mathrm{~N}_{7} \mathrm{O}_{3} \mathrm{~S} \\
(367.43)\end{array}$ & $\begin{array}{c}55 \\
130-132\end{array}$ & $\begin{array}{r}45.71 / \\
45.72\end{array}$ & $\begin{array}{c}5.73 / \\
5.72\end{array}$ & $\begin{array}{r}26.66 / \\
26.67\end{array}$ \\
\hline
\end{tabular}


tassium carbonate and dry acetone led to the formation of ethyl (2-methyl-4-nitro- $1 \mathrm{H}$ imidazol-1-yl) acetate (1). The resulting ester was subjected to hydrazinolysis to yield a solid mass of 1-[2-(hydrazinooxy)-2-oxoethyl]-2-methyl-4-nitro-1H-imidazole (2), which when refluxed with $\mathrm{KOH}$ and carbon disulphide in ethanol gave new 5-[(2-methyl-4-nitro-1H-imidazol-1-yl)methyl]-1,3,4-oxadiazole-2(3H)-thione (3). New Mannich bases $(4 \mathbf{a}-\mathbf{j})$ were obtained by the reaction of 1,3,4-oxadiazole with appropriate amines in the presence of $40 \%$ formaldehyde (Scheme 1) in moderate to good yields.

The newly synthesized compounds were characterized by spectral data and elemental analysis. In the IR spectra of compounds $4 \mathbf{a}-\mathbf{j}$, the absorption band due to the thione $(C=S)$ group was observed at $1100-1150 \mathrm{~cm}^{-1}$. In the ${ }^{1} \mathrm{H}$ NMR spectra of these Mannich bases, the signal due to thione proton around $\delta 13-15$ ppm was absent, indicating the formation of a Mannich base. In a typical example, the ${ }^{1} \mathrm{H}$ NMR spectrum of $4 \mathrm{~d}$ showed a sharp singlet at $\delta 2.26 \mathrm{ppm}$ integrating for three protons of the methyl group of imidazole moiety. The $-\mathrm{N}-\mathrm{CH}_{2}-\mathrm{N}$ - protons appeared at $\delta 5.66 \mathrm{ppm}$ as a singlet integrating for two protons, while the $\mathrm{CH}_{2}$ protons connecting the oxadiazole and imidazole moiety appeared as a singlet at $\delta 5.21 \mathrm{ppm}$ integrating for two protons. The ortho- and meta-protons of $p$-chlorophenyl moiety appeared as two doublets centered at $\delta 7.38$ and $7.67 \mathrm{ppm}$, each integrating for two protons. The imidazole 5-H proton appeared as a singlet at $\delta 8.09 \mathrm{ppm}$ integrating for one proton. The signal due to the $\mathrm{NH}$ proton appeared as a broad singlet at $\delta 10.13 \mathrm{ppm}$ integrating for one proton.

Further evidence for the proposed structure of Mannich bases was obtained by recording their mass spectra. In most cases, the molecular ion peaks were very weak indicating that the molecular ions were not stable (Scheme 2). The fragmented peaks observed in these cases are in conformity with their respective assigned structures. The fragmentation paths for these compounds are shown in Scheme 2. Elemental analysis data together with IR, ${ }^{1} \mathrm{H}$ NMR and MS data supported the proposed structures for compounds 1-3 and 4a-j (Tables I and II).

\section{Structure and antimicrobial activity}

Newly synthesized 3-substituted aminomethyl-5-(2-methyl-4-nitro-1-imidazomethyl)-1,3,4-oxadiazole-2-thiones (4a-j) were examined for antimicrobial activity and the reults are summarized in Table III. The antifungal screening results revealed that Mannich base $4 \mathbf{b}-\mathbf{j}$ exhibited higher to moderate activity against $C$. albicans and T. mentagrophytes compared to standard fluconazole. Compound 4 a with phenyl substituent showed the least activity.

All aminomethyl-5-(2-methyl-4-nitro-1-imidazomethyl)-1,3,4-oxadiazole-2-thiones possessing different substituent groups $(\mathbf{4 b - j})$, except $\mathbf{4 a}$, showed higher inhibitory activity against $C$. albicans than the reference fluconazole. It is worth noting that compound $4 \mathrm{c}$ with $p$-fluorophenyl substituent and $4 \mathbf{f}$ with 2-methyl-4-nitrophenyl substituent showed 5-fold better in vitro activity against C. albicans with MIC 172 and $154 \mu \mathrm{mol} \mathrm{L} \mathrm{L}^{-1}$, respectively, compared to the standard drug fluconazole (MIC $816 \mu \mathrm{mol} \mathrm{L}^{-1}$ ). Besides, compound $4 \mathrm{~h}$ (MIC $368 \mu \mathrm{mol} \mathrm{L}{ }^{-1}$ ) integrating piperazinyl group exhibited 2-fold better activity than the standard drug fluconazole.

Furthermore, the compounds with ethyl, fluoro and bromo groups at para position in phenyl substituent (4b, $4 \mathrm{c}$ and $4 \mathbf{e})$, with 2-methyl-4-nirophenyl substituent (4f), and 
Table II. Spectral data of new compounds

\begin{tabular}{|c|c|c|c|}
\hline Compd. & $\begin{array}{c}\mathrm{IR} \\
\left(v_{\max }, \mathrm{cm}^{-1}\right)\end{array}$ & ${ }^{1} \mathrm{H}$ NMR $(400 \mathrm{MHz}, \delta, \mathrm{ppm})\left(\mathrm{DMSO}-\mathrm{d}_{6}\right)$ & $\begin{array}{c}\text { DART MS } \\
(m / z)\end{array}$ \\
\hline 1 & $\begin{array}{l}2980(\mathrm{CH}), 1728(\mathrm{C}=\mathrm{O}), \\
1533(\mathrm{~N}=\mathrm{C})\end{array}$ & $\begin{array}{l}1.28\left(\mathrm{t}, 3 \mathrm{H}, \mathrm{CH}_{3}\right), 2.34\left(\mathrm{~s}, 3 \mathrm{H}, \mathrm{CH}_{3}\right), 4.22(\mathrm{q}, 2 \mathrm{H} \text {, } \\
\left.\mathrm{CH}_{2}\right), 5.01\left(\mathrm{~s}, 2 \mathrm{H}, \mathrm{N}-\mathrm{CH}_{2}\right), 8.19(\mathrm{~s}, 1 \mathrm{H}, \mathrm{H}-5 \\
\text { imidazole })\end{array}$ & $213(\mathrm{M}+\mathrm{H})^{+}$ \\
\hline 2 & $\begin{array}{l}3151(\mathrm{NH}), 2962(\mathrm{CH}) \\
1686(\mathrm{C}=\mathrm{O}), 1598(\mathrm{~N}=\mathrm{C})\end{array}$ & $\begin{array}{l}2.33\left(\mathrm{~s}, 3 \mathrm{H}, \mathrm{CH}_{3}\right), 4.71\left(\mathrm{~s}, 2 \mathrm{H}, \mathrm{N}-\mathrm{CH}_{2}\right), 5.08(\mathrm{~s}, 2 \mathrm{H}, \\
\left.\mathrm{NH}_{2}\right), 8.18(\mathrm{~s}, 1 \mathrm{H}, \mathrm{H}-5 \text { imidazole }), 9.50(\mathrm{~s}, 1 \mathrm{H}, \\
\mathrm{CONH})\end{array}$ & $199(\mathrm{M}+\mathrm{H})^{+}$ \\
\hline 3 & $\begin{array}{l}3142(\mathrm{NH}), 2952(\mathrm{CH}) \\
1620(\mathrm{~N}=\mathrm{C}), 1135(\mathrm{C}=\mathrm{S})\end{array}$ & $\begin{array}{l}2.40\left(\mathrm{~s}, 3 \mathrm{H}, \mathrm{CH}_{3}\right), 5.38\left(\mathrm{~s}, 2 \mathrm{H}, \mathrm{N}-\mathrm{CH}_{2}\right), 8.16(\mathrm{~s}, 1 \mathrm{H}, \\
\mathrm{H}-5 \text { imidazole }), 14.33(\mathrm{~s}, 1 \mathrm{H}, \mathrm{NH})\end{array}$ & $\begin{array}{l}242(\mathrm{M}+\mathrm{H}, \\
\mathrm{bp})^{+}\end{array}$ \\
\hline $4 a$ & $\begin{array}{l}3151(\mathrm{NH}), 2976(\mathrm{CH}) \\
1595(\mathrm{~N}=\mathrm{C}), 1133(\mathrm{C}=\mathrm{S})\end{array}$ & $\begin{array}{l}2.34\left(\mathrm{~s}, 3 \mathrm{H}, \mathrm{CH}_{3}\right), 5.09\left(\mathrm{~s}, 2 \mathrm{H}, \mathrm{N}-\mathrm{CH}_{2}\right), 5.61(\mathrm{~s}, 2 \mathrm{H}, \\
\left.\mathrm{N}^{-} \mathrm{CH}_{2}-\mathrm{N}\right), 7.25(\mathrm{t}, 1 \mathrm{H}, \mathrm{ArH}), 7.38(\mathrm{t}, 2 \mathrm{H}, \mathrm{ArH}), \\
7.60(\mathrm{~d}, 2 \mathrm{H}, \mathrm{ArH}), 8.04(\mathrm{~s}, 1 \mathrm{H}, \mathrm{H}-5 \text { imidazole }), \\
10.23(\mathrm{~s}, 1 \mathrm{H}, \mathrm{NH})\end{array}$ & $346(\mathrm{M}+\mathrm{H})^{+}$ \\
\hline $4 b$ & $\begin{array}{l}3133(\mathrm{~N}-\mathrm{H}), 2959(\mathrm{C}-\mathrm{H}) \\
1542(\mathrm{~N}=\mathrm{C}), 1139(\mathrm{C}=\mathrm{S})\end{array}$ & $\begin{array}{l}2.33\left(\mathrm{~s}, 3 \mathrm{H}, \mathrm{CH}_{3}\right), 2.51\left(\mathrm{q}, 2 \mathrm{H}, \mathrm{CH}_{2}\right), 2.63(\mathrm{t}, 3 \mathrm{H}, \\
\left.\mathrm{CH}_{3}\right), 5.27\left(\mathrm{~s}, 2 \mathrm{H}, \mathrm{N}-\mathrm{CH}_{2}\right), 5.63\left(\mathrm{~s}, 2 \mathrm{H}, \mathrm{N}-\mathrm{CH}_{2}-\mathrm{N}\right), \\
7.32(\mathrm{~d}, 2 \mathrm{H}, \mathrm{ArH}), 7.60(\mathrm{~d}, 2 \mathrm{H}, \mathrm{ArH}), 8.25(\mathrm{~s}, 1 \mathrm{H}, \\
\mathrm{H}-5 \text { imidazole }), 10.13(\mathrm{~s}, 1 \mathrm{H}, \mathrm{NH})\end{array}$ & $374(\mathrm{M}+\mathrm{H})^{+}$ \\
\hline $4 c$ & $\begin{array}{l}3150(\mathrm{~N}-\mathrm{H}), 2946(\mathrm{C}-\mathrm{H}) \\
1551(\mathrm{~N}=\mathrm{C}), 1133(\mathrm{C}=\mathrm{S})\end{array}$ & $\begin{array}{l}2.33\left(\mathrm{~s}, 3 \mathrm{H}, \mathrm{CH}_{3}\right), 5.11\left(\mathrm{~s}, 2 \mathrm{H}, \mathrm{N}-\mathrm{CH}_{2}\right), 5.66(\mathrm{~s}, 2 \mathrm{H}, \\
\left.\mathrm{N}-\mathrm{CH}_{2}-\mathrm{N}\right), 7.15(\mathrm{t}, 2 \mathrm{H}, \mathrm{ArH}), 7.65(\mathrm{~d}, 2 \mathrm{H}, \mathrm{ArH}), \\
8.11(\mathrm{~s}, 1 \mathrm{H}, \mathrm{H}-5 \text { imidazole) }), 10.78(\mathrm{~s}, 1 \mathrm{H}, \mathrm{NH})\end{array}$ & $365(\mathrm{M}+\mathrm{H})^{+}$ \\
\hline $4 d$ & $\begin{array}{l}3152(\mathrm{~N}-\mathrm{H}), 3049(\mathrm{C}-\mathrm{H}) \\
1539(\mathrm{~N}=\mathrm{C}), 1131(\mathrm{C}=\mathrm{S})\end{array}$ & $\begin{array}{l}2.26\left(\mathrm{~s}, 3 \mathrm{H}, \mathrm{CH}_{3}\right), 5.21\left(\mathrm{~s}, 2 \mathrm{H}, \mathrm{N}-\mathrm{CH}_{2}\right), 5.66(\mathrm{~s}, 2 \mathrm{H}, \\
\left.\mathrm{N}-\mathrm{CH}_{2}-\mathrm{N}\right), 7.38(\mathrm{~d}, 2 \mathrm{H}, \mathrm{ArH}), 7.71(\mathrm{~d}, 2 \mathrm{H}, \mathrm{ArH}), \\
8.09(\mathrm{~s}, 1 \mathrm{H}, \mathrm{H}-5 \text { imidazole }), 10.13(\mathrm{~s}, 1 \mathrm{H}, \mathrm{NH})\end{array}$ & $381(\mathrm{M}+\mathrm{H})^{+}$ \\
\hline $4 e$ & $\begin{array}{l}3132(\mathrm{~N}-\mathrm{H}), 2956(\mathrm{C}-\mathrm{H}) \\
1543(\mathrm{~N}=\mathrm{C}), 1140(\mathrm{C}=\mathrm{S})\end{array}$ & $\begin{array}{l}2.33\left(\mathrm{~s}, 3 \mathrm{H}, \mathrm{CH}_{3}\right), 5.26\left(\mathrm{~s}, 2 \mathrm{H}, \mathrm{N}-\mathrm{CH}_{2}\right), 5.67(\mathrm{~s}, 2 \mathrm{H}, \\
\left.\mathrm{N}^{-} \mathrm{CH}_{2}-\mathrm{N}\right), 7.38(\mathrm{~d}, 2 \mathrm{H}, \mathrm{ArH}), 7.71(\mathrm{~d}, 2 \mathrm{H}, \mathrm{ArH}) \\
8.23(\mathrm{~s}, 1 \mathrm{H}, \mathrm{H}-5 \text { imidazole), } 10.21(\mathrm{~s}, 1 \mathrm{H}, \mathrm{NH})\end{array}$ & $425(\mathrm{M}+\mathrm{H})^{+}$ \\
\hline $4 f$ & $\begin{array}{l}3121(\mathrm{~N}-\mathrm{H}), 3076(\mathrm{C}-\mathrm{H}) \\
1534(\mathrm{~N}=\mathrm{C}), 1147(\mathrm{C}=\mathrm{S})\end{array}$ & 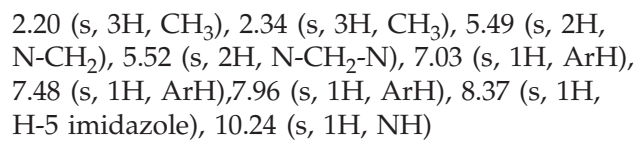 & $405(\mathrm{M}+\mathrm{H})^{+}$ \\
\hline $4 \mathrm{~g}$ & $\begin{array}{l}2940(\mathrm{C}-\mathrm{H}), 1593(\mathrm{~N}=\mathrm{C}) \\
1107(\mathrm{C}=\mathrm{S})\end{array}$ & $\begin{array}{l}2.36\left(\mathrm{~s}, 3 \mathrm{H}, \mathrm{CH}_{3}\right), 5.18\left(\mathrm{~s}, 2 \mathrm{H}, \mathrm{N}-\mathrm{CH}_{2}\right), 5.84(\mathrm{~s}, 2 \mathrm{H}, \\
\left.\mathrm{N}-\mathrm{CH}_{2}-\mathrm{N}\right), 7.20-7.89(\mathrm{~m}, 10 \mathrm{H}, \mathrm{ArH}), 8.05(\mathrm{~s}, 1 \mathrm{H}, \\
\mathrm{H}-5 \text { imidazole })\end{array}$ & 423 \\
\hline $4 \mathrm{~h}$ & $\begin{array}{l}2939(\mathrm{C}-\mathrm{H}), 1596(\mathrm{~N}=\mathrm{C}) \\
1112(\mathrm{C}=\mathrm{S})\end{array}$ & 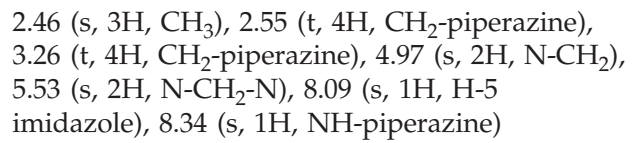 & $339(\mathrm{M}+\mathrm{H})^{+}$ \\
\hline $4 \mathbf{i}$ & $\begin{array}{l}2935(\mathrm{C}-\mathrm{H}), 1595(\mathrm{~N}=\mathrm{C}) \\
1102(\mathrm{C}=\mathrm{S})\end{array}$ & $\begin{array}{l}1.36\left(\mathrm{~s}, 3 \mathrm{H}, \mathrm{CH}_{3}\right), 5.03\left(\mathrm{~s}, 3 \mathrm{H}, \mathrm{NCH}_{3}\right), 2.42(\mathrm{~m}, \\
\left.4 \mathrm{H}, \mathrm{CH}_{2} \text {-piperazine }\right), 2.75\left(\mathrm{~m}, 4 \mathrm{H}, \mathrm{CH}_{2-}\right. \\
\text { piperazine), } 4.97\left(\mathrm{~s}, 2 \mathrm{H}, \mathrm{N}-\mathrm{CH}_{2}\right), 5.53(\mathrm{~s}, 2 \mathrm{H}, \\
\left.\mathrm{N}-\mathrm{CH}_{2}-\mathrm{N}\right), 8.19(\mathrm{~s}, 1 \mathrm{H}, \mathrm{H}-5 \text { imidazole })\end{array}$ & $353(\mathrm{M}+\mathrm{H})^{+}$ \\
\hline $4 \mathbf{j}$ & $\begin{array}{l}2940(\mathrm{C}-\mathrm{H}), 1591(\mathrm{~N}=\mathrm{C}) \\
1116(\mathrm{C}=\mathrm{S})\end{array}$ & $\begin{array}{l}1.37\left(\mathrm{~s}, 3 \mathrm{H}, \mathrm{CH}_{3}\right), 4.90\left(\mathrm{q}, 2 \mathrm{H} \text {, piperazine- }-\mathrm{N}^{-} \mathrm{CH}_{2}\right), \\
1.20\left(\mathrm{t}, 3 \mathrm{H}, \text { piperazine- } \mathrm{CH}_{3}\right), 2.41\left(\mathrm{~m}, 4 \mathrm{H}, \mathrm{CH}_{2}\right. \\
\text {-piperazine }), 2.77\left(\mathrm{~m}, 4 \mathrm{H}, \mathrm{CH}_{2} \text { - piperazine }\right), 4.95 \\
\left(\mathrm{~s}, 2 \mathrm{H}, \mathrm{N}-\mathrm{CH}_{2}\right), 5.53\left(\mathrm{~s}, 2 \mathrm{H}, \mathrm{N}-\mathrm{CH}_{2}-\mathrm{N}\right), 8.18(\mathrm{~s}, \\
1 \mathrm{H}, \mathrm{H}-5 \text { imidazole })\end{array}$ & $368(\mathrm{M}+\mathrm{H})^{+}$ \\
\hline
\end{tabular}


with methyl piperazinyl (4i) or ethyl piperazinyl unit (4j), showed higher activity against T. mentagrophytes (MIC values ranging between $86-340 \mu \mathrm{mol} \mathrm{L}{ }^{-1}$ ) compared to fluconazole (MIC $408 \mu \mathrm{mol} \mathrm{L}{ }^{-1}$ ). Among these, compound 4c exhibited about 5-times better antifungal activity with MIC value $86 \mu \mathrm{mol} \mathrm{L}-1$. Nevertheless, all the tested compounds exhibited weaker activity than fluconazole against T. rubrum. In case of antibacterial activity, almost all the compounds showed weaker activity than

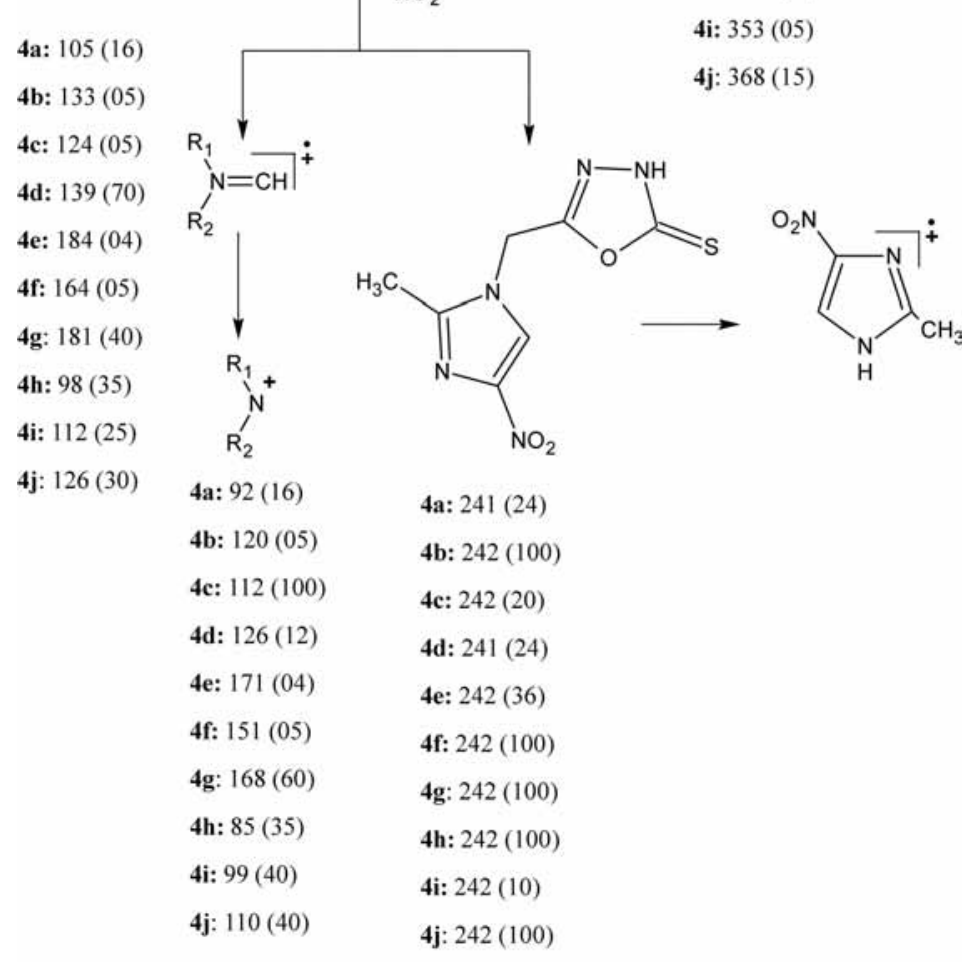

Mass fragmentation pattern

Scheme 2 . 
Table III. Antimicrobial activity of synthesized Mannich bases

\begin{tabular}{|c|c|c|c|c|c|}
\hline \multirow{3}{*}{ Compd. } & \multicolumn{5}{|c|}{ MIC $(\mu \mathrm{mol} \mathrm{L}-1)$} \\
\hline & \multicolumn{3}{|c|}{ Antifungal activity } & \multicolumn{2}{|c|}{ Antibacterial activity } \\
\hline & C. albicans & T. rubrum & T. mentagrophytes & S. pyogenes & S. aureus \\
\hline $4 a$ & 1443 & 1443 & 1443 & 2887 & 1443 \\
\hline $4 b$ & 668 & 334 & 334 & 1335 & 334 \\
\hline $4 c$ & 172 & 343 & 86 & 686 & 343 \\
\hline $4 d$ & 657 & 657 & 1313 & 657 & 1313 \\
\hline $4 e$ & 588 & 294 & 294 & 1175 & 294 \\
\hline $4 \mathrm{f}$ & 154 & 308 & 308 & 308 & 308 \\
\hline $4 \mathrm{~g}$ & 592 & 1184 & 1184 & 592 & 1184 \\
\hline $4 h$ & 368 & 737 & 737 & 368 & 184 \\
\hline $4 i$ & 707 & 354 & 354 & 354 & 354 \\
\hline $4 j$ & 680 & 340 & 340 & 340 & 340 \\
\hline Ciprofloxacin & - & - & - & 94 & 189 \\
\hline Fluconazole & 816 & 200 & 408 & - & - \\
\hline $\begin{array}{l}\text { Solvent control } \\
\text { (DMSO) }\end{array}$ & - & - & - & - & - \\
\hline
\end{tabular}

ciprofloxacin against S. pyogenes and S. aureus. However, piperazinyl substituted compound $4 \mathrm{~h}$ with $\mathrm{MIC}$ value $184 \mu \mathrm{mol} \mathrm{L}^{-1}$ showed promising activity against $S$. aureus compared to the standard antibiotic ciprofloxacin (MIC $189 \mu \mathrm{mol} \mathrm{L}-1)$.

\section{CONCLUSIONS}

A series of new 3-substituted aminomethyl-5-(2-methyl-4-nitro-1-imidazomethyl)-1,3,4-oxadiazole-2-thiones (4a-j) have been synthesized and tested for their antifungal and antimicrobial activities. The results revealed that most of the compounds exhibited moderate to good activity against $C$. albicans, T. mentagrophytes and S. aureus. Compounds $4 \mathrm{~b}-\mathrm{j}$ exhibited excellent antifungal activity against $C$. albicans with low MIC values. In addition, compound $4 \mathrm{c}$ with fluoro substitution exhibited about 5 -fold enhanced activity against T. mentagrophytes compared to fluconazole. The antifungal strength of certain tested compounds was more pronounced than their antibacterial activities. Thus, the promising activities and easy synthesis of Mannich bases carrying imidazole moiety make them very attractive antifungal leads. Further, a complete structure activity relationship and mechanistic approach could contribute to the development of new antimicrobial drugs.

Acknowledgements. - The authors thank Visvesvaraya Technological University, Belgaum, Karnataka, for the financial assistance provided during the course of this research work. Also, the authors are grateful to IIT, Madras, and SAIF, Punjab University, for carrying out elemental analyses, IR, ${ }^{1} \mathrm{H}$ NMR and mass spectra. 


\section{REFERENCES}

1. S. K. Sridhar, S. N. Pandeya, J. P. Stables and A. Ramesh, Anticonvulsant activity of hydrazones, Schiff and Mannich bases of isatin derivatives, Eur. J. Pharm. Sci. 16 (2002) 129-132; DOI: 10.1016/ S0928-0987(02)00077-5.

2. J. R. Dimmock and P. Kumar, Anticancer and cytotoxic properties of Mannich bases, Curr. Med. Chem. 4 (1997) 1-22.

3. A. S. Aboraia, H. M. Abdel-Rahman, N. M. Mahfouz and M. A. El-Gendy, Novel 5-(2-hydroxyphenyl)-3-substituted-2,3-dihydro-1,3,4-oxadiazole-2-thione derivatives: Promising anticancer agents, Bioorg. Med. Chem. 14 (2006) 1236-1246; DOI: 10.1016/j.bmc.2005.09.053.

4. M. A. Ali and M. Shaharyar, Oxadiazole Mannich bases: Synthesis and antimycobacterial activity, Bioorg. Med. Chem. Lett. 17 (2007) 3314-3316; DOI: 10.1016/j.bmcl.2007.04.004.

5. D. Sriram, D. Banerjee and P. Yogeeshwari, Efavirenz Mannich bases: Synthesis, anti-HIV and antitubercular activities, J. Enzyme Inhib. Med. Chem. 24 (2009) 1-5; DOI: 10.1080/14756360701404159.

6. B. M. Kotecka, G. B. Barlin, M. D. Edstein and K. H. Rieckmann, New quinoline di-Mannich base compounds with greater antimalarial activity than chloroquine, amodiaquine or pyronaridine, Antimicrob. Agents Chemother. 41 (1997) 1369-1374.

7. K. V. Sujith, J. N. Rao, P. Shetty and B. Kalluraya, Regioselective reaction: Synthesis and pharmacological study of Mannich bases containing ibuprofen moiety, Eur. J. Med. Chem. 44 (2009) 3697-3702; DOI: 10.1016/j.ejmech.2009.03.044.

8. K. S. Girish, B. Kalluraya, V. Narayana and Padmashree, Synthesis and pharmacological study of 1-acetyl/propyl-3-aryl-5-(5-chloro-3-methyl-1-phenyl-1H-pyrazol-4-yl)-2-pyrazoline, Eur. J. Med. Chem. 45 (2010) 4640-4644; DOI: 10.1016/j.ejmech.2010.07.032.

9. A. M. Isloor, B. Kalluraya and P. Shetty, Regioselective reaction: Synthesis, characterization and pharmacological studies of some new Mannich bases derived from 1,2,4-triazoles, Eur. J. Med. Chem. 44 (2009) 3784-3787; DOI: 10.1016/j.ejmech.2009.04.038.

10. S. Jouneau and J. P. Bazureau, Solvent free aza-annulation using 4-dimethylamino 2-aza-1,3-dienes as $\gamma$-dielectrophiles for a new synthesis of imidazole-4-carboxylates, Tetrahedron Lett. 40 (1999) 8097-8098; DOI: 10.1016/S0040-4039(99)01696-2.

11. L. De Luca, Naturally occurring and synthetic imidazoles: their chemistry and their biological activities, Curr. Med. Chem. 13 (2006) 1-23.

12. M. Boiani and M. González, Imidazole and benzimidazole derivatives as chemotherapeutic agents, Mini Rev. Med. Chem. 5 (2005) 409-424.

13. S. Shadomy and A. Espinel, Manual of Clinical Microbiology, American Society for Microbiology, Washington DC 1980, p. 647. 